\title{
Novel Prognostic Implications of PAQR3 Expression in Breast Cancer after Curative Resection and Systemic Therapy
}

\section{Shiyan Zeng}

Sichuan Cancer Hospital and Institute, University of Electronic Science and Technology of China Sun Lei

Sichuan Cancer Hospital and Institute, University of Electronic Science and Technology of China

\section{Yun Xiao}

Chongqing Traditional Chinese Medicine Hospital

\section{Qian Wang}

The First Affiliated Hospital of Chongqing Medical University

Purong Zhang ( $\sim$ zhangpurong2021@126.com )

Sichuan Cancer Hospital and Institute, University of Electronic Science and Technology of China

\section{Research Article}

Keywords: PAQR3, breast cancer, overall survival, recurrence free survival, ER, PR

Posted Date: April 21st, 2021

DOl: https://doi.org/10.21203/rs.3.rs-383521/v1

License: (c) (i) This work is licensed under a Creative Commons Attribution 4.0 International License. Read Full License 


\section{Abstract}

Background: This study aimed to explore the impact of progestin and adipoQ receptor 3 (PAQR3) on the outcomes for breast cancer patients who received curative resection and systemic therapy.

Methods: The online databases consisting of 2,352 breast cancer patients were used to explore the association between PAQR3 expression and clinicopathological features. Univariate and multivariate survival analysis were performed to identify the survival predictive role of PAQR3. The internal validation strategies were applied.

Results: PAQR3 expression was inversely correlated with estrogen receptor (ER) expression $(P<0.0001)$, progesterone receptor $(P R)$ expression $(P<0.0001)$ and tumor grade $(P<0.0001)$. Higher PAQR3 expression independently predicted shorter overall survival $(O S)$ (hazard ratio $(H R)=1.20,95 \%$ confidence interval $(\mathrm{Cl})=1.06-1.37, \mathrm{P}=0.0055)$ and recurrence free survival $(\mathrm{RFS})(\mathrm{HR}=1.28,95 \% \mathrm{Cl}=1.04-1.45, \mathrm{P}=0.0170)$ in breast cancer patients. In ER+ breast cancer and PR+ breast cancer, PAQR3 was associated with shorter OS (ER+: $\mathrm{HR}=1.28,95 \% \mathrm{Cl}=1.11-1.48, \mathrm{P}=0.0006 ; \mathrm{PR}+\mathrm{HR}=1.30,95 \% \mathrm{Cl}=1.09-1.56, \mathrm{P}=0.0037)$ and $\mathrm{RFS}$ $(E R+: H R=1.46,95 \% \mathrm{Cl}=1.19-1.77, \mathrm{P}=0.0002 ; \mathrm{PR}+\mathrm{HR}=1.48,95 \% \mathrm{Cl}=1.16-1.89, \mathrm{P}=0.0016)$, but no such prognostic values of PAQR3 expression were found in ER- breast cancer and PR-breast cancer.

Conclusions: PAQR3 serves as an independent outcomes predictor for breast cancer.

\section{Introduction}

Breast cancer is the most frequently diagnosed cancer and the leading cause of cancer death among females worldwide [1]. There is an imperative need for the identification of molecular prognostic markers for breast cancer progression.

Progestin and adipoQ receptor (PAQR) superfamily are well known to be activated by the steroid hormones. It could be divided into three subfamilies: adiponectin receptor like proteins, membrane progestin receptor (mPR) containing subfamily, and pore channel forming hemolysins [2,3]. mPR is plasma membrane receptor involved in transducing nongenomic progesterone signaling [4]. mPR structure is predicted including a seven hydrophobic membrane spanning domains, a feature characteristic of GPCRs [5]. In breast cancer, mPR mediates progestin-induced cell survival [6] and progesterone-generated cancer stem cells [7], in a nuclear progesterone receptor independent manner. Romero et al. found that the expression of MPR gamma was elevated in endometrioid and clear cell carcinomas, suggesting a potential role of the MPR in the pathogenesis of tumor [5].

Similar to mPR construction, PAQR3, a seven-transmembrane protein localized to the Golgi apparatus, is also considered regulating GPCR signaling pathway [8]. As previously reported, PAQR3 served as an inhibitor of the ERK activation and suppressor in tumors frequently harboring Ras/Raf mutation, such as melanoma [9], colorectal carcinoma [10], skin cancer [11], gastric cancer [12]. Actually, PAQR3 not only exists as the ERK phosphorylation inhibitor, it is also known as a progestin receptor. Therefore, it may 
have a different role in progestin dependent cancers [13-16]. In this study, we aimed to demonstrate the prognostic value of PAQR3 on the outcomes for breast cancer patients who received curative resection and systemic therapy.

\section{Materials And Methods}

Public online data

The data used in this study was downloaded from GOBO, TCGA and R2 website. The patients were divided into the higher and lower expression of PAQR3 at a specific cut off value (6.56).

Statistics

The paired sample t-test was used to evaluate the difference of PAQR3 expression between tumor and paired normal tissues. The associations between PAQR3 expression and clinicopathological features were evaluated using the $\chi 2$ test. Survival probabilities were calculated by the Kaplan-Meier method, and the log-rank test was used to evaluate statistical difference. The Cox proportional hazards model was used for the multivariate analysis to identify the independent prognostic factors for patients' outcomes. All statistical analyses were conducted using the R program. All tests were two sided, and a $P<0.0500$ was considered significant.

\section{Results}

\section{PAQR3 expression was elevated in human breast cancer}

We compared the PAQR3 expression between breast cancer and paired normal tissues. A decrease in ER and/or PR positive (ER/PR+) tumor tissues was found compared with the paired normal tissues $(\mathrm{P}<$ 0.0500), whereas no such difference was identified in ER and PR negative (ER/PR-) breast cancer ( $P>$ 0.0500) (Fig. 1A). With an online dataset (http://co.bmc.lu.se/gobo) ${ }^{[17]}$, we found PAQR3 expression was negatively associated with ER expression $(P<0.0001)$, whereas positively associated with tumor grade $(P$ $<0.0001$ ) (Fig. 1B).

\section{Paqr3 Expression Was Associated With Breast Cancer Clinicopathological Features}

The associations between PAQR3 expression and clinicopathological features were further explored in 1,873 cases of breast cancer (Cohort A). The demographics and baseline clinicopathological characteristics were listed (Table 1). Tumors with higher levels of PAQR3 were more likely to occur in the patients aged less than 70 years $(P<0.0001)$ and those at the period of premenopause $(P<0.0001)$. In 
addition, PAQR3 expression was associated with the higher tumor grade (III vs. I + II, P<0.0001), lymphatic invasion $(P=0.0170)$, ER loss $(P<0.0001)$ and $P R$ loss $(P<0.0001)($ Table 2 and Fig. 2$)$. 
Table 1

Description of the training cohort characteristics ( $N=1873)$

\begin{tabular}{|c|c|c|c|}
\hline & $\begin{array}{l}\text { Training Set } \\
(n=936)\end{array}$ & $\begin{array}{l}\text { Validation Set } \\
(n=937)\end{array}$ & $\begin{array}{l}\text { Whole Series } \\
(n=1,873)\end{array}$ \\
\hline \multicolumn{4}{|c|}{ Age at diagnosis (years) } \\
\hline$<35$ & $19(2.0)$ & $23(2.4)$ & $42(2.2)$ \\
\hline $35-70$ & $655(70.0)$ & $675(72.0)$ & $1330(71.0)$ \\
\hline$>70$ & $262(28.0)$ & $239(25.6)$ & $501(26.8)$ \\
\hline \multicolumn{4}{|c|}{ Menopausal status } \\
\hline Pre & $199(21.2)$ & $214(22.8)$ & $413(22.0)$ \\
\hline Post & 737 (78.8) & $723(77.2)$ & $1460(78.0)$ \\
\hline \multicolumn{4}{|c|}{ Tumor grade } \\
\hline I & $78(8.3)$ & $79(8.4)$ & $157(8.3)$ \\
\hline ॥ & $384(41.0)$ & $387(41.3)$ & 771 (41.2) \\
\hline III & $474(50.7)$ & $471(50.3)$ & $945(50.5)$ \\
\hline \multicolumn{4}{|c|}{ Tumor stage } \\
\hline T1 & $429(45.8)$ & $404(43.1)$ & $833(44.5)$ \\
\hline T2 & $468(50.0)$ & $474(50.6)$ & $942(50.3)$ \\
\hline T3 & $39(4.2)$ & $59(6.3)$ & $98(5.2)$ \\
\hline \multicolumn{4}{|c|}{ Lymph node status } \\
\hline Negative & $484(51.7)$ & $482(51.4)$ & $966(51.6)$ \\
\hline Positive & $452(48.3)$ & $455(48.6)$ & 907 (48.4) \\
\hline \multicolumn{4}{|l|}{ ER } \\
\hline Negative & $219(23.4)$ & $230(24.5)$ & $449(24.0)$ \\
\hline Positive & $717(76.6)$ & 707 (75.5) & $1424(76.0)$ \\
\hline \multicolumn{4}{|l|}{ PR } \\
\hline Negative & $463(49.5)$ & $435(46.4)$ & $898(48.0)$ \\
\hline Positive & $473(50.5)$ & $502(53.6)$ & $975(52.0)$ \\
\hline
\end{tabular}




\begin{tabular}{|llll|}
\hline & $\begin{array}{l}\text { Training Set } \\
(\boldsymbol{n}=936)\end{array}$ & $\begin{array}{l}\text { Validation Set } \\
(\boldsymbol{n}=937)\end{array}$ & $\begin{array}{l}\text { Whole Series } \\
(\boldsymbol{n}=1,873)\end{array}$ \\
\hline HER2 & & & \\
Negative & $815(87.1)$ & $822(87.8)$ & $1637(87.4)$ \\
Positive & $121(12.9)$ & $115(12.2)$ & $236(12.6)$ \\
Chemotherapy & & & \\
Yes & $201(21.5)$ & $202(21.6)$ & $403(21.5)$ \\
No & $735(78.5)$ & $735(78.4)$ & $1470(78.5)$ \\
\hline Radiotherapy & & & $1135(60.6)$ \\
Yes & $564(60.2)$ & $571(60.9)$ & $738(39.4)$ \\
No & $372(39.8)$ & $366(39.1)$ & $1161(62.0)$ \\
\hline Hormone therapy & & & $712(38.0)$ \\
\hline Yes & $582(62.2)$ & $579(61.8)$ & \\
No & $354(37.8)$ & $358(38.2)$ & \\
\hline
\end{tabular}


Table 2

Comparison of the clinicopathological parameters between low and high PAQR3 expression in training cohort of breast cancer patients $(\mathrm{N}$ $=1,873$ )

\begin{tabular}{|c|c|c|c|}
\hline & $\begin{array}{l}\text { Low PAQR3 } \\
(\mathrm{N}=903)\end{array}$ & $\begin{array}{l}\text { High PAQR3 } \\
(\mathrm{N}=970)\end{array}$ & $\begin{array}{l}P \text { value } \\
\left(\chi^{2} \text { test }\right)\end{array}$ \\
\hline Age at diagnosis (years) & & & $<0.0001$ \\
\hline$<35$ & $12(1.3 \%)$ & $30(3.1 \%)$ & \\
\hline $35-70$ & $579(64.1 \%)$ & $751(77.4 \%)$ & \\
\hline$>70$ & $312(34.6 \%)$ & $189(19.5 \%)$ & \\
\hline Menopausal status & & & $<0.0001$ \\
\hline Pre & $164(18.2 \%)$ & $249(25.7 \%)$ & \\
\hline Post & $739(81.8 \%)$ & $721(74.3 \%)$ & \\
\hline Tumor stage & & & 0.8450 \\
\hline T1 & $399(44.2 \%)$ & $434(44.7 \%)$ & \\
\hline $\mathrm{T} 2+\mathrm{T} 3$ & $504(55.8 \%)$ & $536(55.3 \%)$ & \\
\hline Tumor grade & & & $<0.0001$ \\
\hline $1+\|$ & $535(59.2 \%)$ & $393(40.5 \%)$ & \\
\hline III & $368(40.8 \%)$ & $577(59.5 \%)$ & \\
\hline Lymph node status & & & 0.0170 \\
\hline Negative & $492(54.5 \%)$ & $474(48.9 \%)$ & \\
\hline Positive & $411(45.5 \%)$ & $496(51.1 \%)$ & \\
\hline ER status & & & $<0.0001$ \\
\hline Negative & $113(12.5 \%)$ & $336(34.6 \%)$ & \\
\hline Positive & $790(87.5 \%)$ & $634(65.4 \%)$ & \\
\hline PR status & & & $<0.0001$ \\
\hline Negative & 321 (35.5\%) & $577(59.5 \%)$ & \\
\hline Positive & $582(64.5 \%)$ & $393(40.5 \%)$ & \\
\hline HER2 status & & & 0.0640 \\
\hline Negative & $803(88.9 \%)$ & $834(86.0 \%)$ & \\
\hline Positive & $100(11.1 \%)$ & $136(14.0 \%)$ & \\
\hline
\end{tabular}


Another cohort of 479 breast cancer patients were applied to validate the above findings (Cohort B). The age and baseline characteristics were described (Table S1). Similarly, PAQR3 was associated with the higher tumor grade $(P=0.0080)$, ER loss $(P<0.0001)$ and $P R$ loss $(P=0.0020)$ (Table 3$)$. 
Table 3

Comparison of the clinicopathological parameters between low and high PAQR3 expression in validation cohort of breast cancer patients $(\mathrm{N}=479)$

\begin{tabular}{|c|c|c|c|}
\hline & $\begin{array}{l}\text { Low PAQR3 } \\
(\mathrm{N}=276)\end{array}$ & $\begin{array}{l}\text { High PAQR3 } \\
(N=203)\end{array}$ & $\begin{array}{l}\text { Pvalue } \\
\left(\chi^{2} \text { test }\right)\end{array}$ \\
\hline Age at diagnosis & & & 0.0660 \\
\hline$<35$ & $19(6.9 \%)$ & $19(9.4 \%)$ & \\
\hline $35-70$ & $245(88.8 \%)$ & $182(89.7 \%)$ & \\
\hline$>70$ & $12(4.3 \%)$ & $2(1.0 \%)$ & \\
\hline Tumor stage & & & 0.7280 \\
\hline T1 & $17(6.2 \%)$ & $15(7.4 \%)$ & \\
\hline $\mathrm{T} 2+\mathrm{T} 3$ & $259(93.8 \%)$ & $188(92.6 \%)$ & \\
\hline Lymph node status & & & 0.1960 \\
\hline Negative & $94(34.1 \%)$ & $57(28.1 \%)$ & \\
\hline Positive & $182(65.9 \%)$ & $146(71.9 \%)$ & \\
\hline Tumor grade & & & 0.0080 \\
\hline $1+11$ & $134(48.6 \%)$ & $73(36.0 \%)$ & \\
\hline III & $142(51.4 \%)$ & $130(64.0 \%)$ & \\
\hline AJCC.stage & & & 0.4840 \\
\hline 1 & $4(1.4 \%)$ & $4(2.0 \%)$ & \\
\hline 2 & $159(57.6 \%)$ & $106(52.2 \%)$ & \\
\hline 3 & $113(40.9 \%)$ & $93(45.8 \%)$ & \\
\hline ER status & & & $<0.0001$ \\
\hline Negative & $92(33.3 \%)$ & $113(55.7 \%)$ & \\
\hline Positive & $184(66.7 \%)$ & $90(44.3 \%)$ & \\
\hline PR status & & & 0.0020 \\
\hline Negative & $125(45.3 \%)$ & $122(60.1 \%)$ & \\
\hline Positive & $151(54.7 \%)$ & $81(39.9 \%)$ & \\
\hline HER2 status & & & 0.4360 \\
\hline Negative & 258 (93.5\%) & 194 (95.6\%) & \\
\hline
\end{tabular}




\begin{tabular}{|llll|}
\hline & $\begin{array}{l}\text { Low PAQR3 } \\
(\mathbf{N}=276)\end{array}$ & $\begin{array}{l}\text { High PAQR3 } \\
(\mathbf{N}=203)\end{array}$ & $\begin{array}{l}P \text { value } \\
\left(\mathbf{X}^{2} \text { test }\right)\end{array}$ \\
\hline Positive & $18(6.5 \%)$ & $9(4.4 \%)$ & \\
\hline
\end{tabular}

\section{Paqr3 Independently Predicted The Outcomes For Breast Cancer Patients}

Patients with the higher levels of PAQR3 had a shorter overall survival (OS) $(P=0.0306)$ and recurrence free survival $(R F S)(P<0.0001)$, as compared to the patients with lower PAQR3 expression (Fig. 3).

Univariate survival analysis showed that higher PAQR3 expression, younger age, premenopause, higher tumor grade, lymphatic invasion, ER loss, PR loss, and HER2 overexpression predicted an increased risk of death. When multivariate survival analysis using Cox regression was performed, higher PAQR3 expression remained as the significant predictor for increased risk of death $(\mathrm{HR}=1.20,95 \% \mathrm{Cl}=1.06-$ $1.37, \mathrm{P}=0.0055)$. Other significant predictors included younger age, premenopause, higher tumor grade, lymphatic invasion and HER2 overexpression (Table 4). 
Table 4

Univariate and multivariate analysis of predictive factors for survival in patients with breast cancer

\begin{tabular}{|c|c|c|c|c|c|c|}
\hline \multirow{2}{*}{$\begin{array}{l}\text { Variable } \\
\text { Univariate Analysis }\end{array}$} & \multicolumn{3}{|c|}{ Overall survival } & \multicolumn{3}{|c|}{ Recurrence free survival } \\
\hline & $\mathrm{HR}$ & $95 \% \mathrm{Cl}$ & $P$ value & $\mathrm{HR}$ & $95 \% \mathrm{Cl}$ & $P$ value \\
\hline $\begin{array}{l}\text { PAQR3 } \\
\text { (high/low) }\end{array}$ & 1.14 & $\begin{array}{l}1.01- \\
1.29\end{array}$ & 0.0310 & 1.44 & $\begin{array}{l}1.23- \\
1.69\end{array}$ & $<0.0001$ \\
\hline $\begin{array}{l}\text { Age at diagnosis }(<35 />70 / 35- \\
70)\end{array}$ & 2.08 & $\begin{array}{l}1.84- \\
2.36\end{array}$ & $<.0001$ & - & - & NS \\
\hline $\begin{array}{l}\text { Menopausal status } \\
\text { (Post/Pre) }\end{array}$ & 1.68 & $\begin{array}{l}1.42- \\
1.98\end{array}$ & $\hat{0.0001}$ & - & - & NS \\
\hline $\begin{array}{l}\text { Tumor stage } \\
(\mathrm{T} 2+\mathrm{T} 3 / \mathrm{T} 1)\end{array}$ & - & - & NS & - & - & NS \\
\hline $\begin{array}{l}\text { Tumor grade } \\
(I I I / I+I I)\end{array}$ & 1.34 & $\begin{array}{l}1.18- \\
1.51\end{array}$ & $\hat{0.0001}$ & 1.83 & $\begin{array}{l}1.56- \\
2.15\end{array}$ & $<0.0001$ \\
\hline $\begin{array}{l}\text { Lymph node status } \\
\text { (Positive/Negative) }\end{array}$ & 1.70 & $\begin{array}{l}1.50- \\
1.91\end{array}$ & <. 0001 & 2.28 & $\begin{array}{l}1.94- \\
2.68\end{array}$ & $<0.0001$ \\
\hline $\begin{array}{l}\text { ER status } \\
\text { (Positive/Negative) }\end{array}$ & 0.84 & $\begin{array}{l}0.73- \\
0.96\end{array}$ & 0.0130 & 0.59 & $\begin{array}{l}0.50- \\
0.70\end{array}$ & $<0.0001$ \\
\hline $\begin{array}{l}\text { PR status } \\
\text { (Positive/Negative) }\end{array}$ & 0.76 & $\begin{array}{l}0.68- \\
0.86\end{array}$ & ¿ 0.0001 & 0.61 & $\begin{array}{l}0.52- \\
0.72\end{array}$ & $<0.0001$ \\
\hline $\begin{array}{l}\text { HER2 status } \\
\text { (Positive/Negative) }\end{array}$ & 1.49 & $\begin{array}{l}1.26- \\
1.78\end{array}$ & < 0.0001 & 2.05 & $\begin{array}{l}1.68- \\
2.51\end{array}$ & $<0.0001$ \\
\hline $\begin{array}{l}\text { Chemotherapy } \\
\text { (Yes/No) }\end{array}$ & 1.28 & $1.1-1.48$ & 0.0010 & 2.17 & $\begin{array}{l}1.83- \\
2.58\end{array}$ & $<0.0001$ \\
\hline $\begin{array}{l}\text { Radiotherapy } \\
\text { (Yes/No) }\end{array}$ & 0.85 & $\begin{array}{l}0.75- \\
0.95\end{array}$ & 0.0070 & - & - & NS \\
\hline $\begin{array}{l}\text { Hormone therapy } \\
\text { (Yes/No) }\end{array}$ & 1.25 & $\begin{array}{l}1.10- \\
1.42\end{array}$ & 0.0010 & - & - & NS \\
\hline Multivariable Analysis & $\mathrm{HR}$ & $95 \% \mathrm{Cl}$ & $P$ value & $\mathrm{HR}$ & $95 \% \mathrm{Cl}$ & $P$ value \\
\hline
\end{tabular}

NS, none significance. 


\begin{tabular}{|c|c|c|c|c|c|c|}
\hline Variable & Overa & survival & & Recu & ence fre & ival \\
\hline $\begin{array}{l}\text { PAQR3 } \\
\text { (low/high) }\end{array}$ & 1.20 & $1.06-1.37$ & 0.0055 & 1.28 & $\begin{array}{l}1.04- \\
1.45\end{array}$ & 0.0170 \\
\hline $\begin{array}{l}\text { Age at diagnosis }(<35 / 35- \\
70 />70)\end{array}$ & 2.14 & $1.86-2.46$ & $\begin{array}{l}<.0001 \\
\end{array}$ & - & - & NS \\
\hline $\begin{array}{l}\text { Menopausal status } \\
\text { (Pre/Post) }\end{array}$ & 1.47 & $1.23-1.78$ & $\begin{array}{l}<.0001 \\
0.00\end{array}$ & - & - & NS \\
\hline $\begin{array}{l}\text { Tumor grade } \\
(I+\mid I / I I I)\end{array}$ & 1.25 & $1.10-1.43$ & 0.0010 & 1.41 & $\begin{array}{l}1.18- \\
1.68\end{array}$ & 0.0002 \\
\hline $\begin{array}{l}\text { Lymph node status } \\
\text { (Negative/Positive) }\end{array}$ & 1.61 & $1.39-1.86$ & $\begin{array}{l}<.0001 \\
0.00\end{array}$ & 1.98 & $\begin{array}{l}1.65- \\
2.38\end{array}$ & $<0.0001$ \\
\hline $\begin{array}{l}\text { HER2 status } \\
\text { (Negative/Positive) }\end{array}$ & 1.37 & $1.14-1.65$ & 0.0008 & 1.62 & $\begin{array}{l}1.31- \\
2.01\end{array}$ & $<0.0001$ \\
\hline $\begin{array}{l}\text { Chemotherapy } \\
\text { (Yes/No) }\end{array}$ & 1.28 & $1.04-1.58$ & 0.0194 & - & - & NS \\
\hline $\begin{array}{l}\text { Radiotherapy } \\
\text { (Yes/No) }\end{array}$ & 0.77 & $0.68-0.88$ & $\begin{array}{l}<.0001 \\
0\end{array}$ & - & - & NS \\
\hline
\end{tabular}

Univariate survival analysis showed that higher PAQR3 expression, higher tumor grade, lymphatic invasion, ER loss, PR loss, and HER2 overexpression predicted an increased risk of tumor recurrence. When multivariate survival analysis using Cox regression was applied, higher PAQR3 expression remained as the significant predictor for shorter $\mathrm{RFS}(\mathrm{HR}=1.28,95 \% \mathrm{Cl}=1.04-1.45, \mathrm{P}=0.0170)$. Other significant predictors included higher tumor grade, lymphatic invasion and HER2 overexpression (Table 4).

To verify the predictive role of PAQR3 expression for breast cancer patients' outcomes, we randomly divided 1,873 patients into the training $(\mathrm{N}=936)$ and validation $(\mathrm{N}=937)$ sets. The demographics and baseline clinicopathological characteristics were summarized (Table 1). After adjustment for other predictors including younger age, premenopause, higher tumor grade, lymphatic invasion and HER2 overexpression, the higher PAQR3 expression remained serving as an independent predictor for shorter $O S$ in the training set $(H R=1.24, P=0.0206)$, and was likely to predict the $O S$ in the validation set $(H R=$ $1.18, P=0.0884$ ) (Table S2). However, we did not find that the higher PAQR3 expression predicted shorter RFS in training and validation sets. 


\section{Paqr3 Better Predicted The Outcomes In Er/pr + breast Cancer}

As ER/PR status was associated with PAQR3 expression, we stratified the 1,873 patients according to the ER and PR status, and then studied if PAQR3 expression predicted the outcomes in ER + and PR + breast cancer, respectively.

In ER + breast cancer, multivariate survival analysis using Cox regression showed that PAQR3 independently predicted shorter OS $(\mathrm{HR}=1.28,95 \% \mathrm{Cl}=1.11-1.48, \mathrm{P}=0.0006)$ and $\mathrm{RFS}(\mathrm{HR}=1.46,95 \% \mathrm{Cl}$ $=1.19-1.77, \mathrm{P}=0.0002$ ) (Table 5 and Fig. 4). Nevertheless, no such a predictive role of PAQR3 expression was found in ER- breast cancer. 
Table 5

Multivariate analysis of predictive factors for survival in patients with ER/PR positive breast cancer

\begin{tabular}{|c|c|c|c|c|c|c|c|c|}
\hline \multirow{3}{*}{$\begin{array}{l}\text { Variable } \\
\text { Multivariable Analysis }\end{array}$} & \multicolumn{4}{|c|}{ Overall survival } & \multicolumn{4}{|c|}{ Recurrence free survival } \\
\hline & \multicolumn{2}{|c|}{$\begin{array}{l}\text { ER positive } \\
\text { group }\end{array}$} & \multicolumn{2}{|c|}{$\begin{array}{l}\text { PR positive } \\
\text { group }\end{array}$} & \multicolumn{2}{|c|}{$\begin{array}{l}\text { ER positive } \\
\text { group }\end{array}$} & \multicolumn{2}{|c|}{ PR positive group } \\
\hline & $\mathrm{HR}$ & $\begin{array}{l}P \\
\text { value }\end{array}$ & $\mathrm{HR}$ & $\begin{array}{l}P \\
\text { value }\end{array}$ & $\mathrm{HR}$ & $P$ value & $\mathrm{HR}$ & $\begin{array}{l}P \\
\text { value }\end{array}$ \\
\hline $\begin{array}{l}\text { PAQR3 } \\
\text { (high/low) }\end{array}$ & 1.28 & 0.0006 & 1.30 & 0.0037 & 1.46 & 0.0002 & 1.48 & 0.0016 \\
\hline $\begin{array}{l}\text { Age at diagnosis (< } \\
35 / 35-70 />70)\end{array}$ & 2.21 & $\hat{0.0001}$ & 2.44 & <.0001 & 1.44 & 0.0010 & 1.72 & 0.0001 \\
\hline $\begin{array}{l}\text { Menopausal status } \\
\text { (Post/Pre) }\end{array}$ & 1.75 & $\hat{0} .0001$ & 1.79 & 0.0001 & - & NS & - & NS \\
\hline $\begin{array}{l}\text { Tumor grade } \\
(\mathrm{III} / \mathrm{I}+\mathrm{II})\end{array}$ & 1.25 & 0.0025 & 1.45 & 0.0001 & 1.49 & 0.0001 & 1.75 & $\begin{array}{l}<.0001 \\
0.00\end{array}$ \\
\hline $\begin{array}{l}\text { Lymph node status } \\
\text { (Positive/Negative) }\end{array}$ & 1.58 & $\dot{0} 0001$ & 1.45 & 0.0004 & 2.09 & $\begin{array}{l}<.0001 \\
0\end{array}$ & 2.02 & $\begin{array}{l}<.0001 \\
0.0\end{array}$ \\
\hline $\begin{array}{l}\text { HER2 status } \\
\text { (Positive/Negative) }\end{array}$ & 1.46 & 0.0038 & 1.49 & 0.0327 & 1.85 & 0.0001 & 1.86 & 0.0053 \\
\hline Chemotherapy (Yes/No) & 1.40 & 0.0118 & 1.55 & 0.0092 & 1.45 & 0.0145 & 1.62 & 0.0132 \\
\hline $\begin{array}{l}\text { Radiotherapy } \\
\text { (Yes/No) }\end{array}$ & 0.76 & 0.0002 & 0.71 & 0.0001 & - & NS & - & NS \\
\hline $\begin{array}{l}\text { Hormone therapy } \\
\text { (Yes/No) }\end{array}$ & - & NS & - & NS & 0.772 & 0.0492 & - & NS \\
\hline
\end{tabular}

In PR + breast cancer, PAQR3 also independently predicted shorter OS $(\mathrm{HR}=1.30,95 \% \mathrm{Cl}=1.09-1.56, \mathrm{P}=$ $0.0037)$ and $\mathrm{RFS}(\mathrm{HR}=1.48,95 \% \mathrm{Cl}=1.16-1.89, \mathrm{P}=0.0016)$, as showed in the multivariate survival analysis using Cox regression (Table 5 and Fig. 4). However, no such a role was found for PAQR3 expression in PR- breast cancer.

Internally validating the predictive role of PAQR3 in ER/PR + breast cancer 
We verified the predictive role of PAQR3 expression for breast cancer patients' outcomes according to the ER and PR status. The previously assigned training $(\mathrm{N}=936)$ and validation $(\mathrm{N}=937)$ sets were utilized here.

In ER + breast cancer, PAQR3 consistently served as an independent predictor for $\mathrm{OS}(\mathrm{HR}=1.30, \mathrm{P}=$ 0.0109; $\mathrm{HR}=1.31, \mathrm{P}=0.0106)$ and $\mathrm{RFS}(\mathrm{HR}=1.41, \mathrm{P}=0.0168 ; \mathrm{HR}=1.52, \mathrm{P}=0.0034)$ in both of training and validation sets (Table 6). 
Table 6

Multivariate analysis of predictive factors for survival in ER/PR positive training/validation cohort of breast cancer

\begin{tabular}{|c|c|c|c|c|c|c|c|c|}
\hline \multirow{3}{*}{$\begin{array}{l}\text { ER positive } \\
\text { Multivariable analysis }\end{array}$} & \multicolumn{4}{|c|}{ Overall survival } & \multicolumn{4}{|c|}{ Recurrence free survival } \\
\hline & \multicolumn{2}{|c|}{ training cohort } & \multicolumn{2}{|c|}{$\begin{array}{l}\text { validation } \\
\text { cohort }\end{array}$} & \multicolumn{2}{|c|}{$\begin{array}{l}\text { training } \\
\text { cohort }\end{array}$} & \multicolumn{2}{|c|}{ validation cohort } \\
\hline & $\mathrm{HR}$ & $\begin{array}{l}P \\
\text { value }\end{array}$ & $\mathrm{HR}$ & $\begin{array}{l}P \\
\text { value }\end{array}$ & $\mathrm{HR}$ & $P$ value & $\mathrm{HR}$ & $\begin{array}{l}P \\
\text { value }\end{array}$ \\
\hline $\begin{array}{l}\text { PAQR3 } \\
\text { (high/low) }\end{array}$ & 1.30 & 0.0109 & 1.31 & 0.0106 & 1.41 & 0.0168 & 1.52 & 0.0034 \\
\hline $\begin{array}{l}\text { Age at diagnosis }(<35 / 35- \\
70 />70)\end{array}$ & 1.99 & <. 0001 & 2.38 & <.0001 & 1.37 & 0.0445 & 1.53 & 0.0087 \\
\hline $\begin{array}{l}\text { Menopausal status } \\
\text { (Post/Pre) }\end{array}$ & 1.75 & 0.0014 & 1.69 & 0.0035 & - & NS & - & NS \\
\hline $\begin{array}{l}\text { Tumor grade } \\
(\mathrm{III} / \mathrm{I}+\mathrm{II})\end{array}$ & - & NS & 1.39 & 0.0020 & - & NS & 1.93 & $\begin{array}{l}< \\
0.0001\end{array}$ \\
\hline $\begin{array}{l}\text { Lymph node status } \\
\text { (Positive/Negative) }\end{array}$ & 1.93 & $\begin{array}{l}<.0001 \\
0.00\end{array}$ & 1.31 & 0.0235 & 2.32 & $\begin{array}{l}<.0001 \\
0.0\end{array}$ & 1.88 & 0.0002 \\
\hline $\begin{array}{l}\text { HER2 status } \\
\text { (Positive/Negative) }\end{array}$ & 1.44 & 0.0377 & 1.52 & 0.0360 & 1.84 & 0.0042 & 1.83 & 0.0091 \\
\hline Chemotherapy (Yes/No) & - & NS & 1.66 & 0.0077 & - & NS & 1.62 & 0.0283 \\
\hline $\begin{array}{l}\text { Radiotherapy } \\
\text { (Yes/No) }\end{array}$ & 0.78 & 0.0130 & 0.74 & 0.0045 & - & NS & - & NS \\
\hline & Overa & survival & & & Recur & ence free & rvival & \\
\hline PR positive & traini & g cohort & $\begin{array}{l}\text { valid } \\
\text { coho }\end{array}$ & ion & trainil & cohort & $\begin{array}{l}\text { valid } \\
\text { coho }\end{array}$ & tion \\
\hline Multivariable analysis & $\mathrm{HR}$ & $\begin{array}{l}P \\
\text { value }\end{array}$ & $\mathrm{HR}$ & $\begin{array}{l}P \\
\text { value }\end{array}$ & $\mathrm{HR}$ & $P$ value & $\mathrm{HR}$ & $\begin{array}{l}P \\
\text { value }\end{array}$ \\
\hline $\begin{array}{l}\text { PAQR3 } \\
\text { (high/low) }\end{array}$ & 1.53 & 0.0013 & 1.18 & 0.2101 & 1.91 & 0.0004 & 1.21 & 0.2716 \\
\hline $\begin{array}{l}\text { Age at diagnosis }(<35 / 35- \\
70 />70)\end{array}$ & 2.24 & $\hat{0.0001}$ & 2.39 & $\hat{0} .0001$ & 1.82 & 0.0028 & 1.65 & 0.0075 \\
\hline
\end{tabular}

NS, none significance 


\begin{tabular}{|c|c|c|c|c|c|c|c|c|}
\hline \multirow[b]{2}{*}{$\begin{array}{l}\text { Menopausal status } \\
\text { (Post/Pre) }\end{array}$} & \multicolumn{4}{|c|}{ Overall survival } & \multicolumn{4}{|c|}{ Recurrence free survival } \\
\hline & 1.87 & 0.0027 & 1.65 & 0.0124 & - & NS & - & NS \\
\hline $\begin{array}{l}\text { Tumor grade } \\
(\mathrm{III} / \mathrm{I}+\mathrm{II})\end{array}$ & 1.40 & 0.0105 & 1.56 & 0.0007 & - & NS & 2.24 & $<0.0001$ \\
\hline $\begin{array}{l}\text { Lymph node status } \\
\text { (Positive/Negative) }\end{array}$ & 1.96 & $\begin{array}{l}< \\
0.0001\end{array}$ & - & NS & 2.76 & $\begin{array}{l}<.0001 \\
0.000\end{array}$ & - & NS \\
\hline $\begin{array}{l}\text { HER2 status } \\
\text { (Positive/Negative) }\end{array}$ & - & NS & 2.47 & 0.0005 & - & NS & 2.69 & 0.0006 \\
\hline Chemotherapy (Yes/No) & - & NS & 1.71 & 0.0185 & - & NS & - & NS \\
\hline $\begin{array}{l}\text { Radiotherapy } \\
\text { (Yes/No) }\end{array}$ & 1.96 & 0.0004 & 0.74 & 0.0176 & - & NS & - & NS \\
\hline NS, none significance & & & & & & & & \\
\hline
\end{tabular}

Nevertheless, in PR + breast cancer, the prognostic value of PAQR3 expression could not be internally confirmed (Table 6).

\section{Discussion}

Previous studies have demonstrated the involvement of PAQR3 in various cancers, but its role in breast cancer, whose progression mostly relies on sexual hormone regulation, remains unclear. Chen et al. firstly described that PAQR3 could trap B-Raf to the Glogi apparatus thus inhibit ERK activation [9].

Nevertheless, all previous studies just concentrated on PAQR3's role in ERK inactivation, which they thought contributing to its tumor suppressing role [18]. None considered PAQR3 could also be a potential mediator for the rapid nongenomic hormone actions [4].

It is well known that progestin could increase breast cancer incidence and promote tumor progression through the combination with $\operatorname{mPR}[19,20]$. Moreover, Dressing et al. proposed a potential involvement of $\mathrm{mPR}$ in the progression of breast cancer, since the intracellular signaling initiated by the mPR is broadly similar to that induced in breast cancer growth [21]. mPR was also found to mediate tumor cell survival [6] and cancer stem cells generation [7]. The MPR expression could be regulated by progesterone, one study found an increase in progesterone was shown to coincide with a reduced level of mPR levels [2]. In our study, we found PAQR3 levels were higher in ER-, PR- or poorly differentiated breast cancer, which are well known as the major risk factors for poor prognosis in breast cancer. These suggested that PAQR3 expression distribution in breast cancer differed from other cancers and PAQR3 may possess a promotion role in breast cancer progression. 
In this study, we demonstrated for the first time that higher PAQR3 expression was associated with poor outcomes in breast cancer, especially in ER + breast cancer. Giulianelli et al. found that progestin-induced breast cancer proliferation was dependent on ER, which possibly explained why PAQR3 better predicted the prognosis in ER + breast cancer [15]. The PAQR3's predictive role found in this study was quite different from its role in other cancers, the following points may explain the divergence: 1) Ras/Raf mutation is not common in breast cancer, compared with melanoma and colorectal cancer which possess a high frequency of Ras/Raf mutation [13, 22]; This also means the ERK activation was obvious for breast cancer, thus the ERK inactivation role of PAQR3 was not important in breast cancer; 2) Breast cancer is different from other cancers, it could be regulated by sexual hormones such as progestin, which was recognized as the ligand of PAQR3; 3 ) No such a large cohort of patients was used for prognosis analysis. Therefore, within the findings we mentioned above, we concluded that PAQR3 may possess a promotion role in tumor progression but not occurrence, and its expression might be downregulated by ER and/or PR expression, this could explain why PAQR3 expression decrease could be only found in ER/PR + tumor tissues compared with paired normal tissues.

In summary, to our knowledge, this is the first large sample study of PAQR3's predictive role in breast cancer patients' outcomes. The findings in this study may help understand PAQR3's role in breast cancer, but still requires a further study.

\section{Abbreviations}

PAQR3, Progestin and adipoQ receptor; RKTG,Raf kinase trapping to Golgi;ER, Estrogen receptor; PR, progesterone receptor;mPR,membrane progestin receptor.

\section{Declarations}

Ethics approval and consent to participate

None declared.

Consent for publication

Not applicable.

Availability of data and material

The datasets generated during and/or analysed during the current study are available in the GOBO,TCGA and R2 repositories,(http://co.bmc.lu.se/gobo, https://www.cancer.gov/about-

nci/organization/ccg/research/structural-genomics/tcga, https://hgserver2.amc.nl/cgi-bin/r2/main.cgi).

Competing interest

None declared. 
Acknowledgements

Not applicable.

Funding

Not applicable.

Authors' contributions

Sy.Z and S.L are the experimental designers and the executors of this study, who completed the data analysis and the writing of the first draft of the paper. Y.X participated in experimental design and analysis of experimental results.Pr.Z and Q.W are the authors and the persons in charge of the project, and guided the experimental design, data analysis, paper writing and revision. All authors read and agree on the final text.

\section{References}

1. Torre LA, Bray F, Siegel RL et al. Global cancer statistics, 2012. CA Cancer J Clin 2015; 65:87-108.

2. Fernandes MS, Pierron V, Michalovich D et al. Regulated expression of putative membrane progestin receptor homologues in human endometrium and gestational tissues. J Endocrinol 2005; 187:89101.

3. Tang YT, Hu T, Arterburn M et al. PAQR proteins: a novel membrane receptor family defined by an ancient 7-transmembrane pass motif. J Mol Evol 2005; 61:372-380.

4. Revankar CM, Cimino DF, Sklar LA et al. A transmembrane intracellular estrogen receptor mediates rapid cell signaling. Science 2005; 307:1625-1630.

5. Romero-Sanchez M, Peiper SC, Evans B et al. Expression profile of heptahelical putative membrane progesterone receptors in epithelial ovarian tumors. Hum Pathol 2008; 39:1026-1033.

6. Dressing GE, Alyea R, Pang Y et al. Membrane progesterone receptors (mPRs) mediate progestin induced antimorbidity in breast cancer cells and are expressed in human breast tumors. Horm Cancer 2012; 3:101-112.

7. Vares G, Sai S, Wang B et al. Progesterone generates cancer stem cells through membrane progesterone receptor-triggered signaling in basal-like human mammary cells. Cancer Lett 2015; 362:167-173.

8. Jiang Y, Xie X, Zhang Y et al. Regulation of G-protein signaling by RKTG via sequestration of the G betagamma subunit to the Golgi apparatus. Mol Cell Biol 2010; 30:78-90.

9. Fan F, Feng L, He J et al. RKTG sequesters B-Raf to the Golgi apparatus and inhibits the proliferation and tumorigenicity of human malignant melanoma cells. Carcinogenesis 2008; 29:1157-1163.

10. Wang X, Li X, Fan F et al. PAQR3 Plays a Suppressive Role in the Tumorigenesis of Colorectal Cancers. Carcinogenesis 2012; 33:2228-2235. 
11. Xie X, Zhang Y, Jiang $Y$ et al. Suppressive function of RKTG on chemical carcinogen-induced skin carcinogenesis in mouse. Carcinogenesis 2008; 29:1632-1638.

12. Ling ZQ, Guo W, Lu XX et al. A Golgi-specific protein PAQR3 is closely associated with the progression, metastasis and prognosis of human gastric cancers. Ann Oncol 2014; 25:1363-1372.

13. Davies H, Bignell GR, Cox C et al. Mutations of the BRAF gene in human cancer. Nature 2002; 417:949-954.

14. Hollestelle A, Elstrodt F, Nagel JH et al. Phosphatidylinositol-3-OH kinase or RAS pathway mutations in human breast cancer cell lines. Mol Cancer Res 2007; 5:195-201.

15. Giulianelli S, Vaque JP, Soldati R et al. Estrogen receptor alpha mediates progestin-induced mammary tumor growth by interacting with progesterone receptors at the cyclin D1/MYC promoters. Cancer Res 2012; 72:2416-2427.

16. Wargon V, Riggio $M$, Giulianelli $S$ et al. Progestin and antiprogestin responsiveness in breast cancer is driven by the PRA/PRB ratio via AIB1 or SMRT recruitment to the CCND1 and MYC promoters. Int $J$ Cancer 2015; 136:2680-2692.

17. Ringner M, Fredlund E, Hakkinen J et al. GOBO: gene expression-based outcome for breast cancer online. PLoS One 2011; 6:e17911.

18. Yu X, Li Z, Chan MT et al. PAQR3: a novel tumor suppressor gene. Am J Cancer Res 2015; 5:25622568.

19. Salazar M, Lerma-Ortiz A, Hooks GM et al. Progestin-mediated activation of MAPK and AKT in nuclear progesterone receptor negative breast epithelial cells: The role of membrane progesterone receptors. Gene 2016; 591:6-13.

20. Hanna R, Pang $Y$, Thomas $P$ et al. Cell-surface expression, progestin binding, and rapid nongenomic signaling of zebrafish membrane progestin receptors alpha and beta in transfected cells. $\mathrm{J}$ Endocrinol 2006; 190:247-260.

21. Dressing GE and Thomas $P$ Identification of membrane progestin receptors in human breast cancer cell lines and biopsies and their potential involvement in breast cancer. Steroids 2007; 72:111-116.

22. Hobbs GA, Der CJ, and Rossman KL RAS isoforms and mutations in cancer at a glance. J Cell Sci $2016 ; 129: 1287-1292$.

\section{Figures}


A
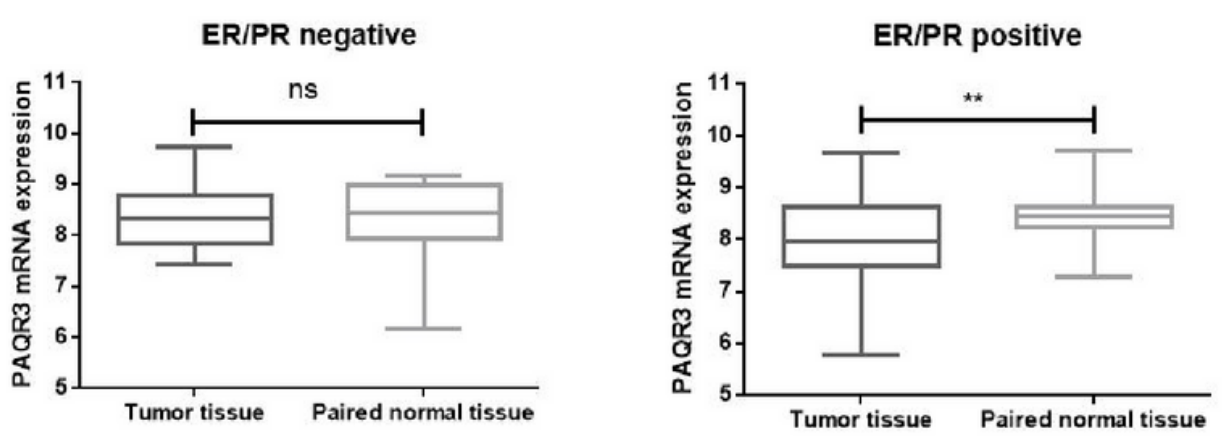

B
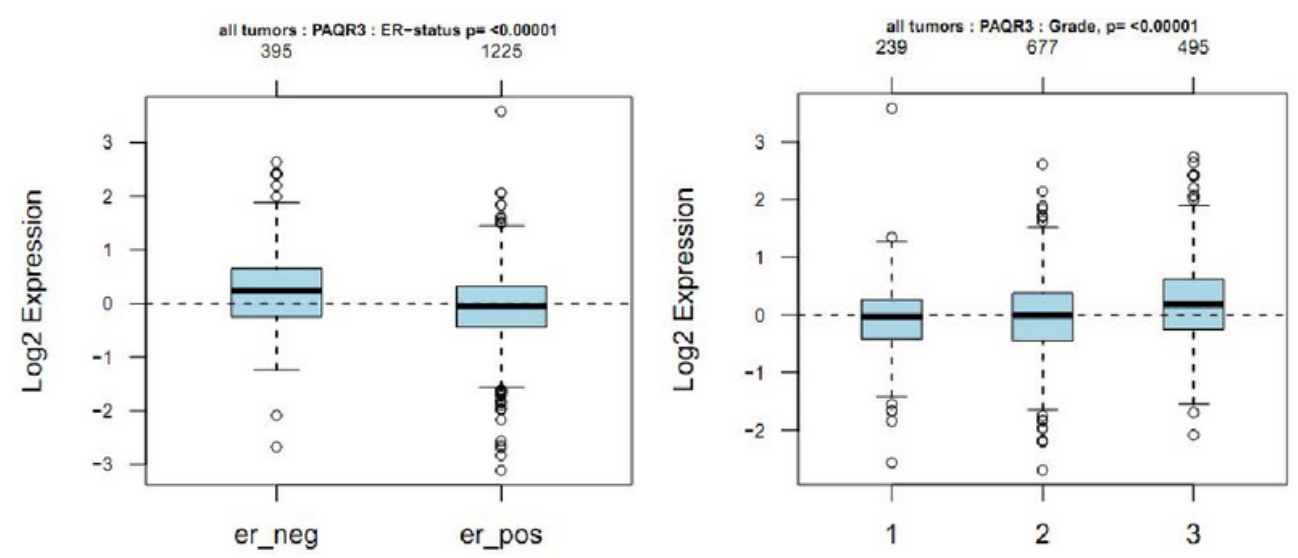

**, $P<0.0100$

ns, none significance

\section{Figure 1}

The PAQR3 expression in human breast cancer. (A) The PAQR3 mRNA expression between tumor and paired normal tissues in ER/PR negative and positive breast cancer, respectively; (B) The PAQR3 mRNA expression between breast cancer with different clinical characteristics (ER status and tumor grade) (**, $\mathrm{P}<0.01)$. 
$\cdots$

$* *, P<0.0100$

ns, none significance
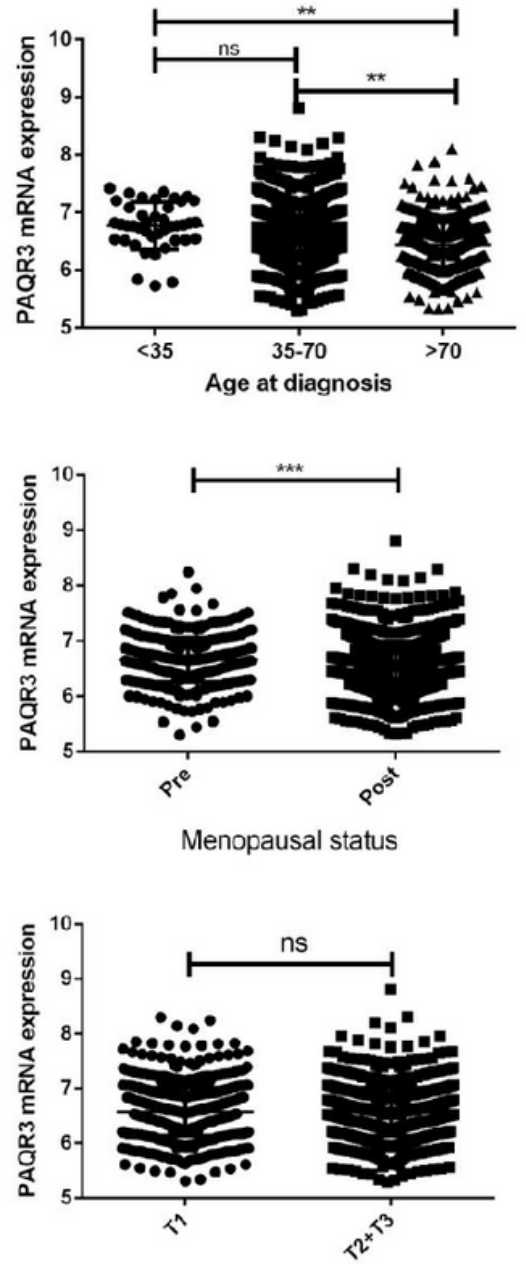

Tumor stage

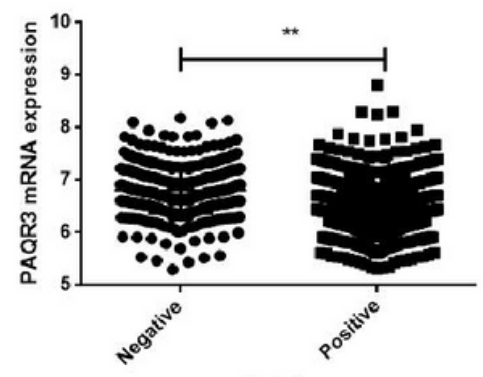

ER status
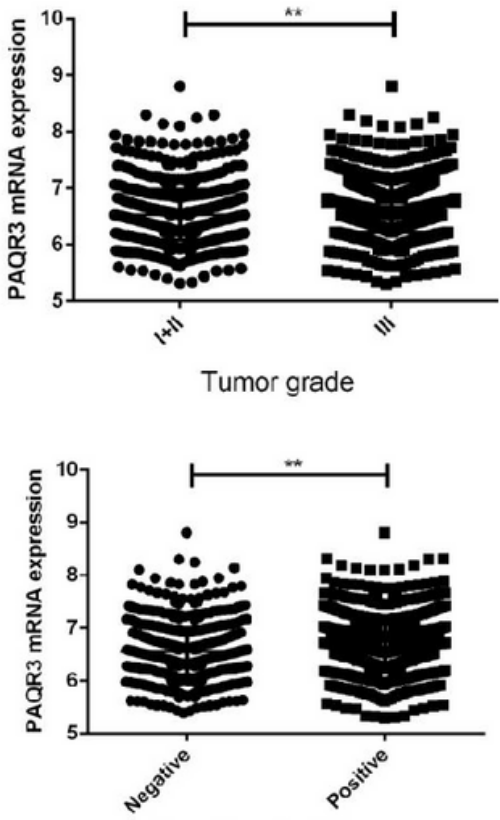

Lymph node status

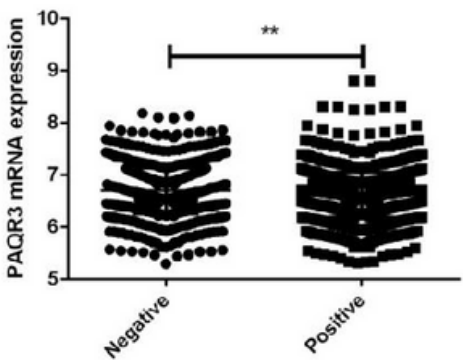

PR status

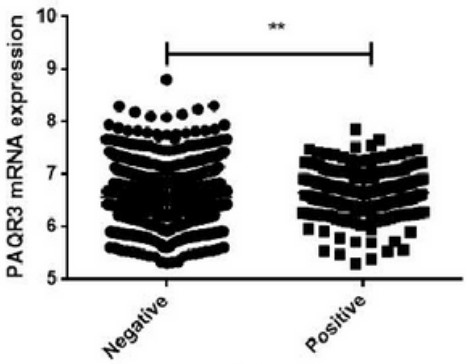

HER2 status

Figure 2

The associations between PAQR3 expression and clinicopathological features. The PAQR3 mRNA expression was associated with age, tumor grade, lymph node status, ER status, PR status and HER2 status (**, $\mathrm{P}<0.01 ; * \star \star, P<0.001)$. 
A

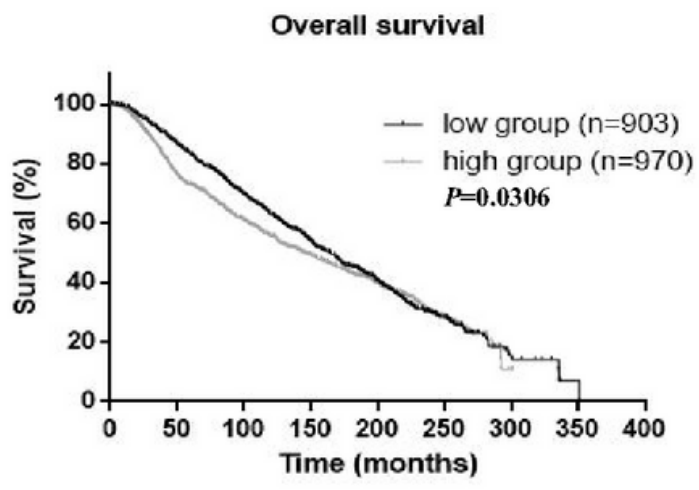

B

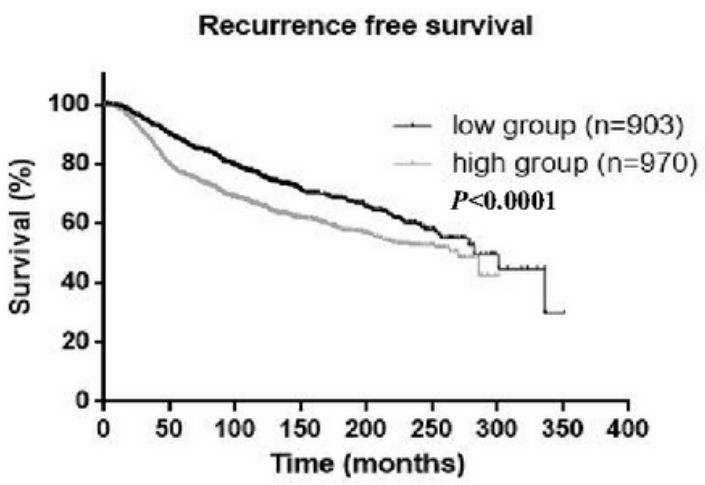

\section{Figure 3}

PAQR3 independently predicted the outcomes for breast cancer patients. Patients with the higher expression of PAQR3 had a shorter overall survival $(O S)(P=0.0306)$ and recurrence free survival (RFS) $(P<0.0001)$. 
Overall survival in ER positive subgroup

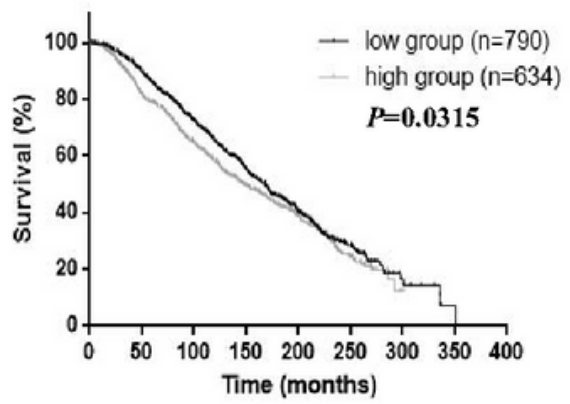

Overall survival in ER negative subgroup

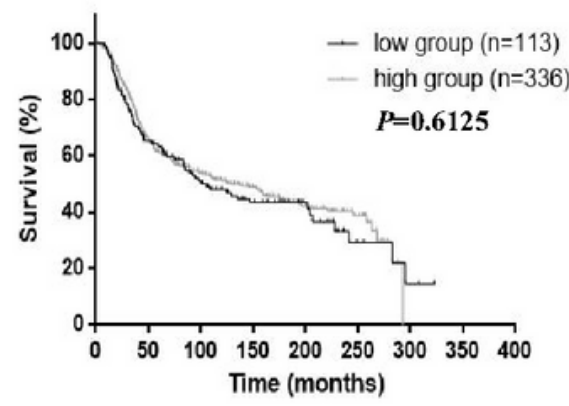

Overall survival in PR positive subgroup

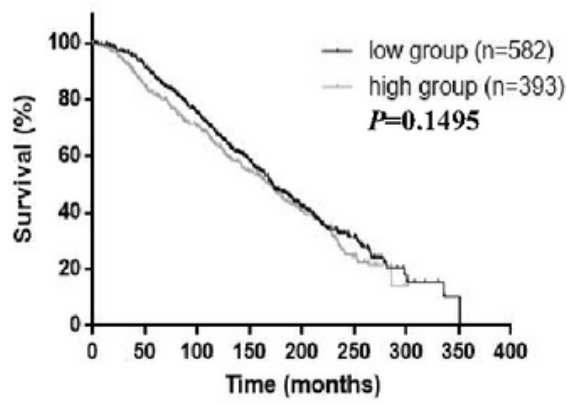

Overall survival in PR negative subgroup

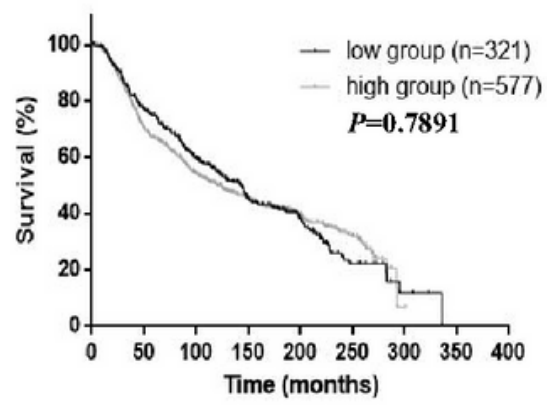

Recurrence free survival in ER positive subgroup

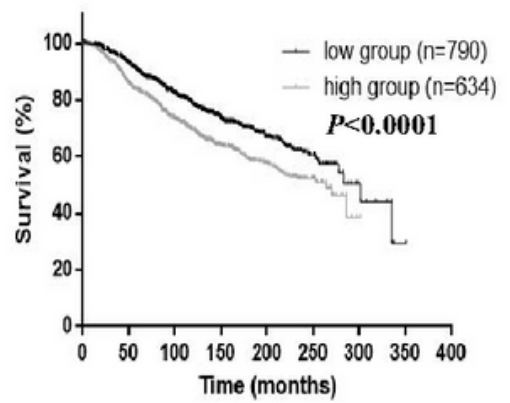

Recurrence free survival in ER negative subgroup

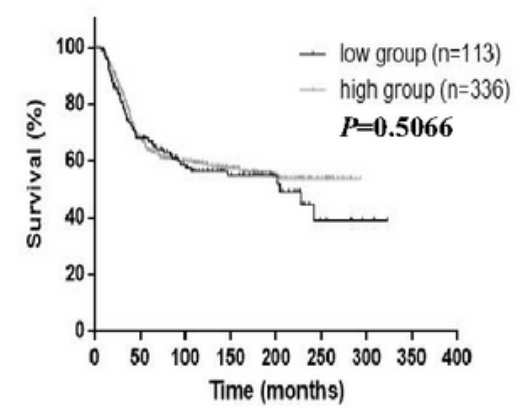

Recurrence free survival in PR positive subgroup

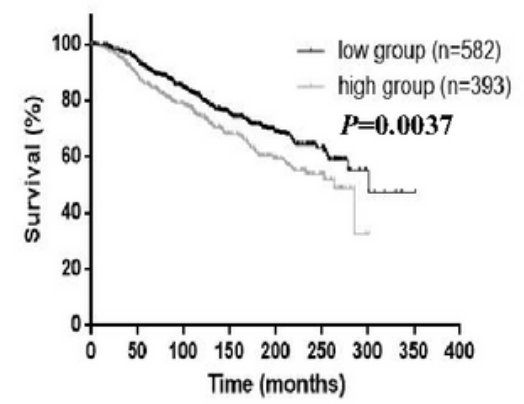

Recurrence free survival in PR negative subgroup

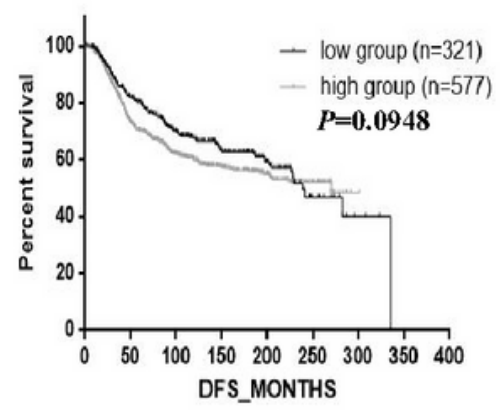

\section{Figure 4}

The prognostic value of PAQR3 in the subgroup analysis. In ER+ breast cancer, PAQR3 independently predicted shorter OS $(P=0.0315)$ and RFS $(P<0.0001)$. In $P R+$ breast cancer, PAQR3 also independently predicted shorter OS $(P=0.1495)$ and RFS $(P=0.0037)$.

\section{Supplementary Files}


This is a list of supplementary files associated with this preprint. Click to download.

- TableS1.doc

- Tables2.doc 